\title{
editorial
}

\section{¿Cómo enfrentarse a una crisis en Salud Pública?}

\begin{abstract}
$\mathscr{L}$ a pérdida de la salud ha sido siempre una de las principales preocupaciones del ser humano ${ }^{1}$. Aunque las circunstancias de nuestra sociedad actual sean muy diferentes a las que se daban en otras épocas, en las que predominaban grandes epidemias que diezmaban a la población, sin embargo, la capacidad y la velocidad con la que se transmite la información de unos lugares a otros en nuestra época hace que problemas sanitarios localizados se comporten, en ocasiones, como verdaderas crisis en salud pública.

Los grandes avances científicos en el conocimiento de la etiología y patogenia de las enfermedades no han supuesto un descenso en la aparición de nuevas entidades clínicas o en el resurgimiento de otras ya conocidas. Por el contrario, en los últimos años estamos asistiendo a un fenómeno caracterizado por la aparición de nuevas enfermedades, enfermedades conocidas que vuelven a resurgir y exposiciones medioambientales que ponen en riesgo la salud de todos.

Más recientemente, la amenaza de riesgos asociados a ataques bioterroristas resurge con fuerza y nos hacen pensar en enfermedades de las que no hemos tenido la oportunidad de ver casos durante nuestra vida profesional y que sólo hemos estudiado de manera superficial en nuestro periodo de formación en la facultad.

Todo este panorama forma un entorno idóneo para que cualquier problema sanitario alcance dimensiones imprevisibles, y a su vez hagan que su control sea un problema para los profesionales que trabajan en el marco de la Salud Pública².
\end{abstract}

\section{DEFINICIÓN DE CRISIS}

De un modo operativo, se puede definir un contexto de crisis como una situación que cumple tres características principales: punto de cambio o tendencia de una situación preestablecida; situación aguda o subaguda; necesidad de actuación urgente.

Este conjunto puede aplicarse a muchas circunstancias, pero como es obvio este comentario estará relacionado con los problemas de salud o de riesgo potencial de los ciudadanos a enfermar.

\section{SITUACIÓN DE CRISIS. DIAGNÓSTICO}

Uno de los mayores problemas contra los que se enfrenta un profesional médico es una situación de crisis. Generalmente, la falta de pre- 
paración, lo inesperado del problema, la incertidumbre y la presión social, y de los medios de comunicación, derivan en actuaciones descoordinadas y desacertadas.

El diagnóstico de una crisis potencial puede establecerse en base a una de las siguientes señales de alarma: tamaño (número de enfermos, extensión de la exposición, alta letalidad). Gravedad de los casos (en términos de severidad clínica de los casos, tipo de exposición, impacto que pueda tener el "outcome" en la población, etc.). Capacidad de difu sión de la información (influencia de los medios de comunicación, tipo de medios y tiempo de dedicación de los mismos a la noticia). Siempre que haya otras implicaciones asociadas (ejemplo: políticas, judiciales, etc). Ataques deliberados con generación de miedo (caso de los ataques de bioterrorismo).

En la práctica, este tipo de situaciones no son excesivamente frecuentes y las crisis son controlables desde su inicio debido al conocimiento previo que se suele tener de las mismas. Sin embargo, una vez desencadenado un problema sanitario de envergadura, existen una serie de circunstancias o características que conviene conocer y que van a ser definitivas en la evolución de las crisis: desbordamiento y desorganización; confusión sobre los métodos de estudio (prioridades); predominio de las contradicciones en la comunicación, algunas de ellas con segundas intenciones no controlables con facilidad; alteraciones en la percepción del riesgo por parte de la población e incluso entre los técnicos; adopción de medidas no siempre contrastadas científicamente y con cierta carga de falta de credibilidad; aparente disolución en el tiempo; aislamiento del problema, con el paso del tiempo, a un grupo reducido de la población de los que nadie se acuerda una vez pasada la crisis.

\section{TIPOS DE CRISIS}

Aunque sea de un modo operativo, es interesante comentar algunos aspectos de este tipo de problemas. No todas las crisis se comportan del mismo modo, por ejemplo, los problemas que comienzan a partir de una exposición tienen algunos aspectos diferentes en su mecánica de funcionamiento si los comparamos con aquéllos que comienzan por la aparición de un síndrome clínico. En los primeros, la mayor presión va a venir ejercida por la opinión pública, que se verá canalizada rápidamente a través de los medios políticos y de comunicación, derivando el gran peso de la crisis hacia los expertos del área encargada de medir dicha exposición (químicos, biólogos, etc.). Por el contrario, un brote de una enfermedad vendrá a recaer sobre los profesionales clínicos y, sólo en ocasiones, en los responsables del área de la salud pública. Este pequeño matiz de presentación supone, además de diferentes enfoques técnicos, presiones externas que dificultan el manejo y que conviene conocer para afrontar debidamente. 
En España tenemos ejemplos de crisis de los dos tipos ${ }^{3,4}$ y lo mismo ocurre en otros países ${ }^{5,6}$. No obstante, el mayor problema siempre ha consistido, en todas las partes donde se ha producido un problema de este tipo, en el manejo y control de una situación en la que necesariamente tienen que intervenir numerosos agentes sociales interactuando con los profesionales de la salud.

En estos momentos los ataques bioterroristas suponen la mayor preocupación de las sociedades occidentales. No porque sean por sí mismos el mayor riesgo de enfermar al que podemos estar expuestos, sino porque la influencia de la incertidumbre, el miedo y la presión mediática lo ponen al frente de la actualidad en salud. Por su propia naturaleza, los ataques bioterroristas, se escapan de nuestro control desde los primeros momentos, ya que no se rigen por las leyes naturales de distribución de enfermedades infecciosas o por las vías de exposición a los tóxicos más habituales. En caso de un ataque bioterrorista, el sujeto que produce y diseña el ataque tratará de introducir el problema en la población de manera que pase desapercibido el máximo tiempo posible, hasta que se produzca una gran onda epidémica de una enfermedad poco tratable. La única vía de actuación en este supuesto, para llegar al diagnóstico de manera rápida, es la de aumentar la formación de los profesionales médicos de primer nivel sobre aquellos agentes más sospechosos y establecer unos canales muy específicos de actuación con cadenas de responsabilidad predeterminadas. El entrenamiento combinado con la información son las únicas soluciones posibles para mitigar los efectos?

\section{OBJETIVOS EN SALUD PÚBLICA}

Desde un punto de vista de la salud pública lo que nos interesa es controlar inmediatamente la exposición o interrumpirla en su caso, a la vez que se actúa sobre el huésped intentando modificar su respuesta. Sin embargo, es interesante remitirnos a la definición de riesgo para poder comprender, el por qué este tipo de situaciones se descontrolan.

Entendemos por riesgo la probabilidad de que, bajo determinadas circunstancias, una situación concreta produzca daño. Esto implica dos conceptos: a) probabilidad de que ocurra un evento adverso y b) conse cuencias de dicho evento adverso.

Frente a estos dos conceptos "Probabilidad de que ocurra" y "Consecuencias" aparece la capacidad que la sociedad, y más concretamente los individuos que en ella habitan, tienen para percibir y manejar dicha situación. Es bien conocido que la percepción de los riesgos por parte de los sujetos, incluso de muchos profesionales, no guarda correlación con el riesgo real en términos de riesgo relativo o en términos de consecuencias hacia la salud. Es por ello por lo que otros interlocutores implicados (medios de comunicación, administradores, políticos, sistemas judiciales, etc.) ${ }^{9}$ con capacidad de intervención producen, sino son bien canalizados, más distorsión de esta percepción que a su vez actúa de re- 
troalimentación del problema, dificultando el manejo del mismo hasta límites a veces insospechables.

Existen numerosos esquemas y líneas guías de manejos de este tipo de situaciones, pero las que tienen más probabilidad de éxito son aquéllas que consideran que el problema no es sólo técnico y dan participación a todas las partes implicadas en el proceso para que, a través del diálogo, se puedan llegar a acuerdos que tengan soporte científico y que faciliten la transmisión de las decisiones a la población sin generar ansiedad. La toma de decisiones en estos casos tiene un carácter participativo y facilita la credibilidad de los argumentos técnicos ${ }^{10}$.

\section{OTROS PUNTOS A TENER EN CONSIDERACIÓN}

Por último hay dos puntos importantes a resaltar en el enfoque de una crisis: a) establecer un proceso de evaluación y b) seguimiento de los enfermos.

El proceso de evaluación de las decisiones adoptadas parece ser algo obvio, pero en realidad pocos grupos piensan en ello o, si lo hacen, no establecen los principios necesarios para su desarrollo. El proceso de evaluación de las decisiones debe ser adoptado como algo intrínseco del manejo de la crisis desde el comienzo de la misma. No deben existir dudas al respecto, aunque esto suponga inversiones extras, una vez pasados los primeros momentos. Los efectos beneficiosos de este proceso son: establecimiento de criterios de evaluación (definición de éxito); asegura la credibilidad de la evaluación y de los evaluadores; determina si ha habido éxito; identifica las lecciones que hay que aprender; identifica las lagunas; determina si el coste-beneficio de la decisión fue razonable.

- Seguimiento del problema de salud. Además de la evaluación de las decisiones, los problemas de salud derivados del evento que ha desencadenado la crisis, independientemente de su intensidad y duración, deben considerarse como susceptibles de seguimiento clínico general. Lo habitual es que una vez desaparecida la situación de crisis todo el mundo se olvide del problema, de los enfermos y/o expuestos, quedando sin vigilancia posterior. No es posible dar normas sobre este punto ya que dependerá del tipo de problema que estemos tratando, pero sí se puede establecer, como recomendación general, la creación de un registro de expuestos y/o enfermos y su seguimiento. El periodo de seguimiento y los objetivos del mismo se deben decidir en función de la información que se tenga del problema en cada caso.

\section{CONCLUSIONES}

Resolver una crisis que afecta a la salud de los ciudadanos no es sólo un problema técnico. Supone un entramado complejo de presiones e in- 
fluencias que el profesional debe conocer de antemano y comprender para poder desarrollar su trabajo en libertad, única manera de conseguir que lo realizado sirva para algo y no quede como una actividad más.

Conocer los métodos de estudio aplicables en caso de crisis es muy relevante, pero organizarse, saber colaborar con los implicados, asesorar a otras partes que lo demanden y conocer los mecanismos que operan en este tipo de situaciones forman parte de los conocimientos que los profesionales que se dedican al cuidado de la salud de todos los ciudadanos deben conocer en profundidad.

La realidad actual, nos enfrenta a una posibilidad de una ataque bioterrorista, que sin duda y caso de producirse, será generadora de una gran crisis. Aunque la probabilidad parezca baja, la inquietud por conocer este tipo de riesgos debe formar parte de nuestra iniciativa y por lo tanto debemos incrementar nuestra formación al respecto, refrescando conocimientos o adquiriéndolos en los múltiples portales de internet que se nos ofrecen ${ }^{11}$. Sólo un diagnóstico precoz podría mitigar los efectos de un posible ataque.

M. Posada de la Paz Investigador en Temas de Salud Pública. Madrid

\section{BIBLIOGRAFÍA}

1. Posada M. Diet and food contaminants. In: Steenland K, Savitz DA, eds. Topics in Environmental Epidemiology. New York: Oxford University Press, 1997; 64-88.

2. Bertollini R, Lebowitz M, Saracci R, Savitz D. Environmental Epidemiology. Exposure and Disease. Denmark: CRC. Lewis Publishers, 1996.

3. World Health Organization. Toxic Oil Syndrome. Current knowledge and futures perspectives. WHO Regional Publications. European Series, 42. England, 1992.

4. Antó JM, Sunyer J, Reed CE, Sabrià J, Martínez F, Codina R, et al. Preventing asthma epidemics due to soybeans by dust control measures. N Engl J Med 1993; 329: 1760-3.

5. O'Brien KL, Selaniko JD, Hecdivert Ch, Placide MF, Louis M, Barr DB, et al. Epidemic of pediatric deaths from acute renal failure caused by diethylene glycol poisoning. JAMA 1998; 279 (15): 1175-80.

6. Centers for Disease Control and Prevention. Deaths among children during an outbreak of hand, foot, and mouth disease-Taiwan, Republic of China, April-July 1998. MMWR 1998; 47 (30); 629-32.

7. Khan Ali S, Levitt Alexandra M, Sage Michael J. Biological and Chemical Terrorism: Strategic Plan for Preparedness and Response. Recomendation of the CDC Strategic Planning Workgroup. MMWR Recomendations and Reports. April, 21 2000; (49) RR-4. http://cisat.isciii.es/mmwr/pdf/rr/rr4904.pdf 
8. World Health Organization. Assesing the health Consequences of Major Chemical Incidents-Epidemiological Approaches. WHO Regional Publications. Denmark: European Series, 79, 1997.

9. Philip CR, Gray Richard M. Stern and Marco Biocca. Communicating about risks to environment and health in Europe. Published on behalf of the World Health Organization Regional Office for Europe by Kluwer Academic Publishers. Netherlands, 1997.

10. Omenn GS, Kessler AC, Anderson NT, Chiu PY, Doull J. The presidential/Congressional Commission on Risk Assesment and Risk Mana gement. Framwork for Environmental health Risk Management. Washington, 1997.

11. CDC. Public Health Emergency Preparedness \& Response. http:// www.bt.cdc.gov

Nota del autor: "Las opiniones vertidas en este artículo son responsabilidad únicamente del autor, y no reflejan necesariamente la opinión de la institución a la que está afiliado". 